\title{
Photon-aided and photon-inhibited Tunneling of Photons
}

\author{
Xuele Liu* and G.S. Agarwal \\ Department of Physics, Oklahoma State University, Stillwater, Oklahoma 74078, USA
}

(Dated: August 10, 2021)

\begin{abstract}
In light of the interest in the transport of single photons in arrays of waveguides, fiber couplers, photonic crystals, etc., we consider the quantum mechanical process of the tunneling of photons through evanescently or otherwise coupled structures. We specifically examine the issue of tunneling between two structures when one structure already contains few photons. We demonstrate the possibility of both photon-aided and photon-inhibited tunneling of photons. The bosonic nature of photons enhances the tunneling probability. We also show how the multiphoton tunneling probability can be either enhanced or inhibited due to the presence of photons. We find similar results for higher-order tunneling. Finally, we show that the presence of a squeezed field changes the nature of tunneling considerably.
\end{abstract}

PACS numbers: 42.82.Et, 42.50.-p, 05.60.Gg

\section{INTRODUCTION}

The quantum transport properties of fermions have been extensively studied in the last century $[1,2]$. One interesting phenomenon in quantum transport of fermions is the Coulomb blockade effect [3], which happens when electrons are transported through a quantum dot. The Coulomb blockade effect, arising from the Pauli exclusion principle, inhibits tunneling by the presence of electrons in the quantum dot. The differences between energy levels in small quantum dots are very large (with the unoccupied higher level far from the Fermi energy); therefore, the transport probability is very small and the tunneling is blocked.

An analog of the Coulomb blockade $[4,5]$ was demonstrated by Birnbaum et al. [6], showing that if an atom resonant with a strongly coupled single mode cavity could absorb one photon, then the absorption of a second photon was inhibited. A similar experiment was reported in the context of superconducting qubits [7]. Another phenomenon is the dipole blockade [8] in Rydberg atoms where the excitation of a second atom to a Rydberg state is forbidden if one atom is already excited to a Rydberg state. The first excitation makes the second excitation non-resonant which leads to the blockade effect, and is similar to the Coulomb blockade in that it is based on the energy gap.

The inhibited tunneling of photons that we discuss in this paper has a more fundamental origin - the bosonic statistics of photons, just like the Fermi statistics in the Coulomb blockade. To understand this, we examine the photon tunneling between two coupled single modes, with each mode referring to a different structure. This can be realized by coupled single-mode waveguide devices [Fig. 1(a)] [9, 10], fiber couplers, coupled resonators, and optomechanical systems[11]. The waveguides coupled by evanescent fields can be tailored by changing the distance

\footnotetext{
*xuele@okstate.edu
}

between waveguides $[9,10]$. The evanescent coupling is responsible for the tunneling of photons. This is equivalent to tunneling between two potential wells [Fig. 1(b)]; the tunneling that we discuss is a coherent process.

In this paper we report photon-aided tunneling (PAT) and photon-inhibited tunneling (PIT), which can occur due to the presence of photons in the other waveguide. We find that even when the energy gap between the two waveguides is large and the single-photon tunneling is negligible, the PAT is significant and becomes about $1 / \mathrm{e}$ when the number of photons in the other waveguide is large. PIT occurs when the energy gap is small. When the energy gap is zero, the tunneling rate without any photons in the other waveguide is $100 \%$; tunneling is totally inhibited when photons are present in the other waveguide. Both PAT and PIT depend on the bosonic nature of the photons. They are not allowed for electrons due to the Fermi statistics. Our results are exact and go far beyond the perturbation theory.

\section{MODEL AND METHOD}

Without loss of generality, we discuss the tunneling of photons between two coupled single modes in the two waveguides labeled $A$ and $B$ [Fig. 1(a)], which can be realized using silica on silicon $[9,10]$. Arrays of waveguides have been extensively studied with both classical and quantum light $[9,10]$. We can also use fiber couplers. We start with the simplest possibility that the waveguide $A$ contains one photon and the waveguide $B$ contains $n$ photons; we calculate the probability $P_{(1, n) \rightarrow(0, n+1)}$ of the one photon from the waveguide $A$ tunneling to the waveguide $B$. If $P_{(1, n) \rightarrow(0, n+1)}>P_{(1,0) \rightarrow(0,1)}$, then we conclude that the presence of $n$ photons in waveguide $B$ enhances tunneling. Experimentally, use of an avalanche photodiode to detect the probability of having zero photons in waveguide $A$ gives us a measurement of the tunneling of a single photon to the waveguide $B$. Note that efficient sources of single heralding photons are available [12]. Theoretically, the Hamiltonian for a system of two 


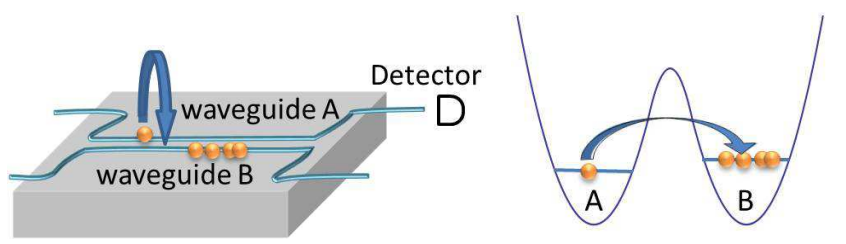

FIG. 1: (Color online) (a) Silica-on-silicon coupled waveguides (see, for example, [9]). The detector in the dark state at time $t$ signals the tunneling from $A$ to $B$. (b) Tunneling from potential well $A$ to potential well $B$, with the presence of bosons at well $B$.

coupled waveguides is given by

$$
\hat{H}=\hbar \Delta\left(a^{\dagger} a-b^{\dagger} b\right)+\hbar J\left(a^{\dagger} b+b^{\dagger} a\right) ;
$$

Here $a, a^{\dagger}\left(b, b^{\dagger}\right)$ are the annihilation and creation operators for the field in $A(B)$. Generally, the two waveguides $A$ and $B$ have different refractive indices leading to different detunings $\varepsilon_{0} \pm \hbar \Delta$. Here $\varepsilon_{0}$ gives an overall phase $e^{-\mathrm{i} \varepsilon_{0} t}$, which does not affect the transport probability. Thus, we set $\varepsilon_{0}=0$. The refractive index leads to a phase shift of the field but does not change the frequency of the photon. We consider one-dimensional propagation along the length of the wave guide. The coupling constant $J$ is the tunneling energy of the photon needed to go from one waveguide to the other. It depends on the distance between the two waveguides, and can be calculated from the overlap of the electric field distributions [13]. Its value, say, for two silicon waveguides at a distance aprat of $4 \mu \mathrm{m}$ is about $0.51 \mathrm{~mm}^{-1}$ in units of $\frac{c}{n}$ [9]. Other waveguides like AlGaAs have parameters in a similar range [10].

The Hamiltonian (1) can be diagonalized by writing it in terms of the angular momentum operators and then by using the properties of the rotation group. However, in considerations of the dynamics we have found an alternative and elegant procedure by using the Heisenberg equations of motion and hence we do not give the exact eigenfunctions of (1). If the system is initially in the state $|\Psi(0)\rangle=|n, m\rangle$, i.e., there are $n$ photons in the waveguide $A$ and $m$ photons in the waveguide $B$, then at time $t$ (which is proportional to the propagation distance) the system is in the state

$$
|\Psi(t)\rangle=\frac{\left(a^{\dagger}(-t)\right)^{n}\left(b^{\dagger}(-t)\right)^{m}}{\sqrt{n ! m !}}|0,0\rangle .
$$

Equations (2) can be proved as follows. We write the intial state as

$$
|\Psi(0)\rangle=|n, m\rangle=\frac{\left(a^{\dagger}\right)^{n}\left(b^{\dagger}\right)^{m}}{\sqrt{n ! m !}}|0,0\rangle .
$$

Let $U(t)$ be the time evolution operator, then

$$
\begin{aligned}
& |\Psi(t)\rangle=U(t)|n, m\rangle \\
= & \frac{U(t)\left(a^{\dagger}\right)^{n} U^{\dagger}(t) U(t)\left(b^{\dagger}\right)^{m} U^{\dagger}(t)}{\sqrt{n ! m !}} U(t)|0,0\rangle,
\end{aligned}
$$

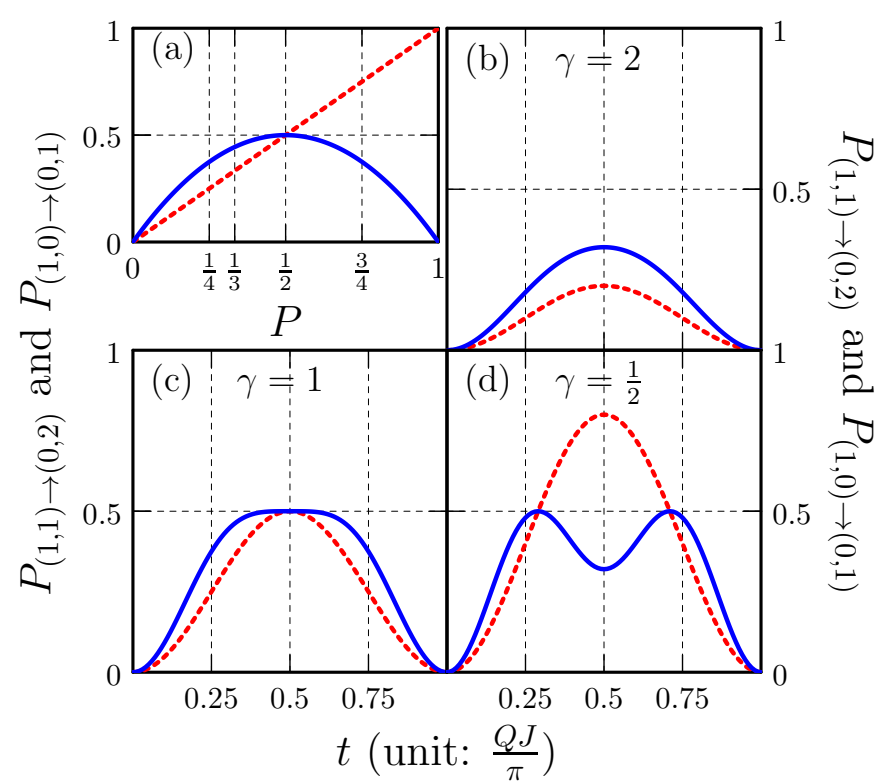

FIG. 2: (Color online) The tunneling probabilities $P_{(1,0) \rightarrow(0,1)}$ (red dashed) and $P_{(1,1) \rightarrow(0,2)}$ (blue solid) as functions of (a) $P$; (b)-(d) scaled time $\frac{Q J t}{\pi}$ for different values of $\gamma=\frac{\Delta}{J}$.

which, on noticing that $|0,0\rangle$ is an eigenstate of $H$ with zero energy $U(t)|0,0\rangle=|0,0\rangle$, reduces to (2) with $a^{\dagger}(-t)=U(t) a^{\dagger} U^{\dagger}(t)$, etc. The time dependent operators $a^{\dagger}(-t)$ and $b^{\dagger}(-t)$ can be obtained through the Heisenberg equations of motion. We quote the result:

$$
\left[\begin{array}{c}
a^{\dagger}(-t) \\
b^{\dagger}(-t)
\end{array}\right]=\left[\begin{array}{cc}
\sqrt{1-P} e^{-\mathrm{i} \theta} & -\mathrm{i} \sqrt{P} \\
-\mathrm{i} \sqrt{P} & \sqrt{1-P} e^{\mathrm{i} \theta}
\end{array}\right]\left[\begin{array}{c}
a^{\dagger}(0) \\
b^{\dagger}(0)
\end{array}\right] .
$$

On defining $\gamma=\frac{\Delta}{J}, Q=\sqrt{1+\gamma^{2}}$, and $P_{0}=\frac{1}{Q^{2}}$, the amplitude of the off-diagonal term is given by $\sqrt{P}$ with

$$
P=P_{0} \sin ^{2}(Q J t) .
$$

The amplitude of diagonal term is given by $\sqrt{1-P}$, and the corresponding phase $\theta$ can be calculated from the probability $\sqrt{1-P} e^{-\mathrm{i} \theta}=\cos (Q J t)-\mathrm{i} \frac{\gamma}{Q} \sin (Q J t)$.

At time $t$, the probability that the system is in the state $\left|n^{\prime}, m^{\prime}\right\rangle$ is given by $P_{(n, m) \rightarrow\left(n^{\prime}, m^{\prime}\right)}=\left|\left\langle n^{\prime}, m^{\prime} \mid \Psi(t)\right\rangle\right|^{2}$. We can then use $P_{(n, m) \rightarrow\left(n^{\prime}, m^{\prime}\right)}$ as the time dependent tunneling probability of $\left(n-n^{\prime}\right)$ photons from waveguide $A$ to waveguide $B$. The tunneling that we discuss is reversible and is like Josephson tunneling [14]. In fact formula (6) is similar to the relation in the context of Josephson tunneling. We would like to emphasize that the tunneling discussed here is between two subsystems that are finite in the tunneling direction.

\section{ONE-PHOTON TUNNELING}

If the system is initially in the state $\Psi(0)=|1, n\rangle$, i.e., one photon in waveguide $A$ and $n$ photons in waveguide 
$B$, then the tunneling probability of the one photon from $A$ to $B$ is given by

$$
P_{(1, n) \rightarrow(0, n+1)}=(n+1)(1-P)^{n} P .
$$

The standard case of tunneling is a special case of Eq.(7) when there is no photon in waveguide $B$, i.e., $n=0$; then $P_{(1,0) \rightarrow(0,1)} \equiv P$. We note that the quantity $P$ is like the well-known Rabi oscillation in the populations of a two-level system in an external field. This is because if there is only one photon in the problem, then the state space involves only two levels $|1,0\rangle$ and $|0,1\rangle$. Notice that this one-particle tunneling probability $P$ is the same as in the fermion case. In fact, we can see that, when $\Delta$ is large compared to $J$, then the energy gap between the two modes is large (which means a high barrier at the junction), leading to a large $\gamma$ and a small tunneling probability. It should be mentioned that back tunneling $P_{(1, n) \rightarrow(1+m, n-m)}$ with $0<m \leqslant n$ exists, but we do not need it as we set the experiment to detect no photon in $A$.

In order to illustrate how the presence of photons within the other waveguide affects the tunneling process, we first compare the tunneling probability $P_{(1,1) \rightarrow(0,2)}$ in the presence of one photon in the other waveguide with $P$. From Fig. 2(a), we can see that when $P \leq 1 / 2$, $P_{(1,1) \rightarrow(0,2)}>P$, i.e., the presence of one photon in the waveguide $B$ enhances the probability of tunneling, and PAT occurs. It is easy to show from Eq.(7) that the maximum difference $\left(P_{(1,1) \rightarrow(0,2)}-P\right)_{\max }=1 / 9$ is reached when $P=1 / 3$. We now show that PAT occurs for any range of values of $\Delta$ and $J$. When the gap $2 \Delta$ is such that $\gamma=\frac{\Delta}{J} \geq 1$, we have $P_{0}<1 / 2, P<1 / 2$, and PAT always occurs. This is shown in Fig. 2.(b). Here $\gamma=2$ and $P_{(1,1) \rightarrow(0,2)} \geq P$ for any time $t$. When the energy gap is small $\gamma \leq 1$ and thus $P_{0} \geq 1 / 2$, we can still observe PAT. The oscillation structure of $P$, Eq. (6) guarantees that there exist time regions such that $P \leq 1 / 2$ so that $P_{(1,1) \rightarrow(0,2)} \geq P$. [Figs. 2(c) and 2(d)].

From Fig. 2(a), we also observe the appearance of photon-inhibited tunneling when $P>1 / 2$. In particular, when $P=1(100 \%$ tunneling probability without the presence of any photon in waveguide $B$ ), we have exactly $P_{(1,1) \rightarrow(0,2)}=0$, and the photon tunneling is totally inhibited. PIT can be observed only when the gap is small, $\gamma<1$, so that $P_{0}>1 / 2$, which allows $P>1 / 2$ in some time region [Fig. 2(d)].

The existence of PAT and PIT shows a competition mechanism introduced by the bosonic nature of photons, as is seen from Eq. (7). Two factors are multiplied with $P$ : the first one $(n+1) \geq 1$ is an aided term, which makes it possible for $P_{(1, n) \rightarrow(0, n+1)}$ to be bigger than $P$. It comes from the bosonic nature that $a^{\dagger}|n\rangle=\sqrt{n+1}|n+1\rangle$. The bosonic nature shows that if a state contains more bosons, then it is easier to add an extra boson to it. The second term $(1-P)^{n} \leq 1$ is an inhibited term, which reflects the tendency that the $n$ photons from waveguide $B$ stay in waveguide $B$. Notice that $(1-P)$ gives the probability that the one

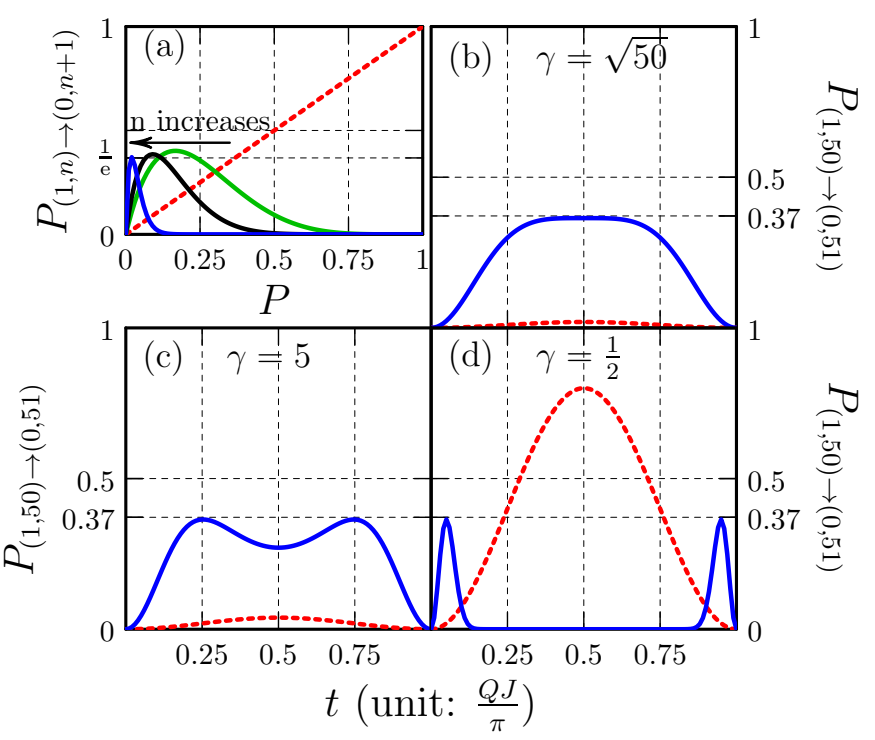

FIG. 3: (Color online) (a) The tunneling probability $P_{(1, n) \rightarrow(0, n+1)}$ as a function of $P$ for $n=0$ (red dashed); $n=5$ (green); $n=10$ (black); $n=50$ (blue). (b)-(d) The time dependence of the tunneling probability $P_{(1,50) \rightarrow(0,51)}$ (blue) as compared to that of $P_{(1,0) \rightarrow(0,1)}$ (red dashed) for different values of $\gamma$.

photon still wants to stay at its own site. The power $n$ reflects the probability of the $n$-photon state remaining an $n$-photon state. When $n$ is larger, this term becomes smaller. The aided term $(n+1)$ is fixed when the photon number is fixed; while the inhibited term $(1-P)^{n}$ can decrease from 1 to 0 when $P$ increases from 0 to 1 . As a result: if $P$ is small, the aided term dominates and we observe PAT; if $P$ is large, then we observe PIT.

We next examine $P_{(1,1) \rightarrow(0,2)}$. When $P<1 / 2$, the tunneling probability is small; every photon wants to stay in its own state, and the inhibited term $(1-P)^{n}$ is large. At this time the aided term $(n+1)$ is important, leading to $P_{(1,1) \rightarrow(0,2)}>P$. When $P>1 / 2$, the inhibited term suppresses the positive effect of the aided term $(n+1)$.

We now discuss the general case of the tunneling probability $P_{(1, n) \rightarrow(0, n+1)}$ of one photon tunneling in the presence of $n$ photons. From Fig. 3(a), we can see that when the number of photons is increased, the region of PAT becomes smaller and occurs at smaller values of $P$; however, the maximum value does not decrease very much. In fact, from Eq. (7), we get $\left(P_{(1, n) \rightarrow(0, n+1)}\right)_{\max }=\left(1-\frac{1}{n+1}\right)^{n}$ when $P=1 /(n+1)$. When the number of photons in waveguide $B$ is very large, $n \rightarrow \infty$, we can get $\left(P_{(1, n) \rightarrow(0, n+1)}\right)_{\max } \rightarrow 1 / \mathrm{e} \simeq 0.37$. Therefore we get very significant PAT especially when $\Delta$ is large, which leads to very small values of $P$. We compare the tunneling probability $P_{(1, n) \rightarrow(0, n+1)}$ with $P$ for different $\gamma$. In Fig. $3(\mathrm{~b})$, when $\gamma=\sqrt{n}=\sqrt{50}$ so that $P_{0}=1 / 51$, we can see that the tunneling without any photons in waveguide $B$ is very small, while $P_{(1,50) \rightarrow(0,51)}$ has a plateau at 0.37 for a large time range; In Fig. 3(c), the aided tunneling 


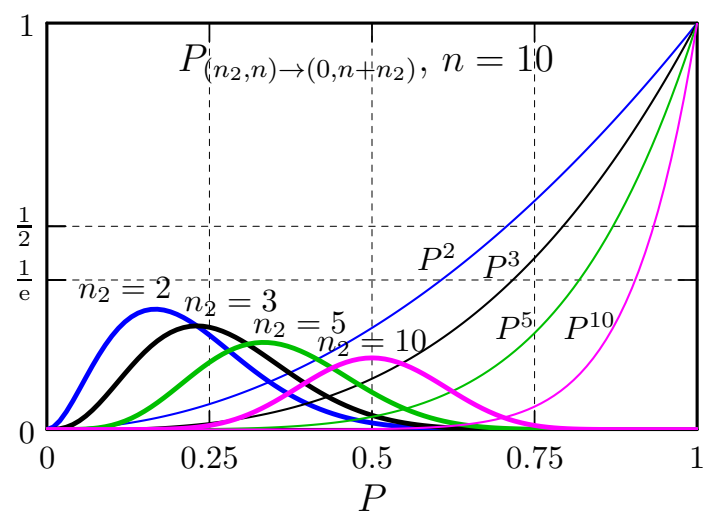

FIG. 4: $P_{\left(n_{2}, n\right) \rightarrow\left(0, n+n_{2}\right)}$ (thick lines), the multiphoton tunneling probability in presence of 10 photons in the waveguide $B$, as a function of $P$ for $n_{2}=2,3,5,10$. The multiphoton tunneling probabilities without presence of photons in the waveguide $B, P_{\left(n_{2}, 0\right) \rightarrow\left(0, n_{2}\right)}=P^{n_{2}}$ are given by thin lines.

is still significant for $\gamma=5$. In Fig. 3(d), when the gap is small, the aided tunneling acts as a pulse in the vicinity of time period $T$. This means that even for a large gap $\Delta$, we can always find a finite photon tunneling probability $\left(P_{(1, n) \rightarrow(0, n+1)}\right)_{\max }$ near $\frac{1}{\mathrm{e}}$ by choosing $n \geq \gamma^{2}=\frac{\Delta^{2}}{J^{2}}$, whereas for large $\Delta, P_{(1,0) \rightarrow(0,1)}$ is negligible.

\section{MULTI-PHOTON TUNNELING}

The above PAT can be generalized to multi-photon tunneling. In Fig. 4, we compare $P_{\left(n_{2}, n\right) \rightarrow\left(0, n+n_{2}\right)}$ with $P_{\left(n_{2}, 0\right) \rightarrow\left(0, n_{2}\right)}=P^{n_{2}}$. Obviously, $P_{\left(n_{2}, 0\right) \rightarrow\left(0, n_{2}\right)}$ decreases when $n_{2}$ increases since $P<1$. This is intuitive in that it is harder for more photons to tunnel to another waveguide. We can see that when $n_{2}=10$, the probability $P_{\left(n_{2}, 0\right) \rightarrow\left(0, n_{2}\right)}$ is close to zero for most values of $P$. However, with photons in waveguide $B$, the tunneling is significant. We can always find a finite maximum of $P_{\left(n_{2}, n\right) \rightarrow\left(0, n+n_{2}\right)}$. If $n \gg n_{2}$, this tunneling probability is even greater than the one-photon tunneling $P_{(1,0) \rightarrow(0,1)}$ as shown in Fig. 4.

Using Eq. (2), we find the result for $P_{\left(n_{2}, n\right) \rightarrow\left(0, n+n_{2}\right)}$ to be:

$$
P_{\left(n_{2}, n\right) \rightarrow\left(0, n+n_{2}\right)}=\left(\begin{array}{c}
n+n_{2} \\
n_{2}
\end{array}\right)(1-P)^{n} P^{n_{2}} .
$$

where $\left(\begin{array}{c}n \\ m\end{array}\right)=\frac{n !}{(n-m) ! m !}$ is the binomial coefficient. From this equation, we can clearly see that the inhibited term is still given by $(1-P)^{n}$, while the aided term is changed to $\left(\begin{array}{c}n+n_{2} \\ n_{2}\end{array}\right)$. This means that aided tunneling should be more important when $n_{2}$ increases. However, noticing that $P_{\left(n_{2}, 0\right) \rightarrow\left(0, n_{2}\right)}$ decreases, the absolute value $P_{\left(n_{2}, n\right) \rightarrow\left(0, n+n_{2}\right)}$ may still decrease. This can be seen from Fig. 4 which shows the decreasing peak of $P_{\left(n_{2}, n\right) \rightarrow\left(0, n+n_{2}\right)}$ with the increase of $n_{2}$. The maximum peak value is given by

$$
\left(P_{\left(n_{2}, n\right) \rightarrow\left(0, n+n_{2}\right)}\right)_{\max }=\left(\begin{array}{c}
n+n_{2} \\
n_{2}
\end{array}\right) \frac{n_{2}^{n_{2}} n^{n}}{\left(n+n_{2}\right)^{n+n_{2}}} .
$$

which is reached when $P=\frac{n_{2}}{n+n_{2}}$. The limit when $n \rightarrow \infty$, is the maximum of $\frac{1}{n_{2} !}\left(\frac{n_{2}}{\mathrm{e}}\right)^{n_{2}}$. It decreases when $n_{2}$ increases. However, even for $n_{2}=10$, we still have the finite tunneling at about $12.5 \%$, which is much greater than the corresponding tunneling probability $P_{(10,0) \rightarrow(0,10)}=\left(\frac{1}{20}\right)^{10}$ in the absence of any photons in the waveguide $B$.

\section{THE FIELD IN A COHERENT STATE AND A SQUEEZED STATE}

In an experiment, it is much easier to prepare the field in a coherent state than in a state with fixed photon number. The coherent state $|\beta\rangle=e^{-|\beta|^{2} / 2} \sum_{n=0}^{\infty} \frac{\beta^{n}}{\sqrt{n !}}|n\rangle$ has the average photon number $\bar{n}=\left\langle\beta\left|b^{\dagger} b\right| \beta\right\rangle=|\beta|^{2}$. We may then discuss the possibility of PAT and PIT with the field in a coherent state in the waveguide $B$ with a fixed average photon number $\bar{n}$. The probability $P_{\left(n_{2} ; \beta\right) \rightarrow\left(0 ; \beta, n_{2}\right)}$ of $n_{2}$ photons tunneling from waveguide $A$ to waveguide $B$ is given by (see the Appendix)

$$
P_{\left(n_{2} ; \beta\right) \rightarrow\left(0 ; \beta, n_{2}\right)}=e^{-\bar{n}}{ }_{1} F_{1}\left[1+n_{2}, 1, \bar{n}(1-P)\right] P^{n_{2}},
$$

where the confluent hypergeometric function ${ }_{1} F_{1}[a, b ; z]$ is defined by

$$
{ }_{1} F_{1}[a, b ; z]=\sum_{k-0}^{\infty} \frac{(a)_{k}}{(b)_{k}} \frac{z^{k}}{k !}
$$

here $(a)_{k}$ and $(b)_{k}$ are Pochhammer symbols, given by $(a)_{0}=1$ and $(a)_{k}=a(a+1) \cdots(a+k-1)$ for $k>1$.

In the special case of one-photon tunneling, the result is rather simple:

$$
P_{(1 ; \beta) \rightarrow(0 ; \beta, 1)}=e^{-\bar{n} P}[1+\bar{n}(1-P)] P .
$$

The formulas (10) and (12) are complicated; it is not easy to separate aided and inhibited terms. However, from Fig. 5, we observe that, the results for the coherent state case are very similar to those for the case of a Fock state in waveguide $B$, especially when $\bar{n}=n$ is large. We examine the maximum of $P_{(1 ; \beta) \rightarrow(0 ; \beta, 1)}$, which is $\frac{\sqrt{\bar{n}^{2}+2 \bar{n}+5}-2}{\bar{n}} \mathrm{e}^{\frac{1}{2}\left(\sqrt{\bar{n}^{2}+2 \bar{n}+5}-\bar{n}-3\right)}$, and occurs for $P=\frac{\bar{n}^{2}+3 \bar{n}-\bar{n} \sqrt{\bar{n}^{2}+2 \bar{n}+5}}{2 \bar{n}^{2}}$. For large $\bar{n}$, $\lim _{\bar{n} \rightarrow \infty}\left(P_{(1 ; \beta) \rightarrow(0 ; \beta, 1)}\right)_{\max }=\frac{1}{\mathrm{e}}$, which is the same as for the Fock state.

The tunneling probabilities are also sensitive to the photon statistics of photons in waveguide $B$. To illustrate this, we consider the field in a squeezed state 


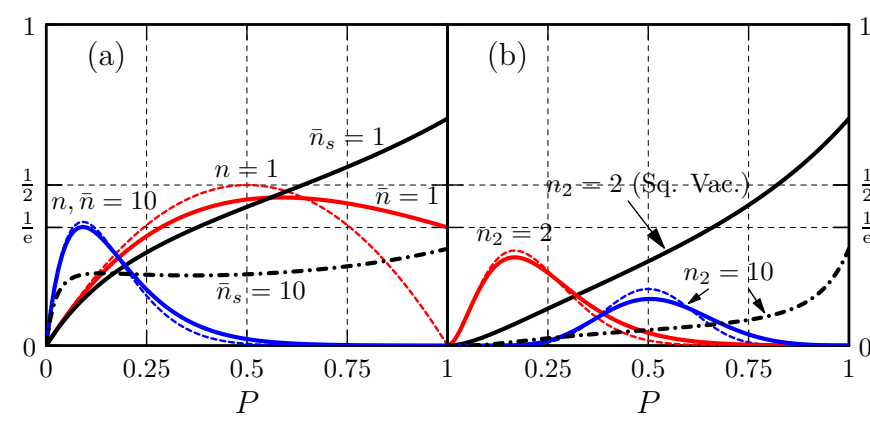

FIG. 5: (Color online) Comparison of photon tunneling probabilities with the field in waveguide $B$ in a coherent state and in a Fock state. (a) The single-photon tunneling probability $P_{(1 ; \beta) \rightarrow(0 ; \beta, 1)}$ (solid lines) and $P_{(1 ; n) \rightarrow(0 ; n+1)}$ (dashed lines) as a function of $P$; (b) The multiphoton tunneling probability $P_{\left(n_{2} ; \beta\right) \rightarrow\left(0 ; \beta, n_{2}\right)}$ (solid lines) and $P_{\left(n_{2}, n\right) \rightarrow\left(0, n+n_{2}\right)}$ (dashed lines) in the presence of (on average) ten photons in waveguide $B$, as a function of $P$. The black solid and dash-dotted lines represent the tunneling probability for a field in squeezed vacuum in waveguide $B$.

$|\xi\rangle=\frac{1}{\sqrt{\cosh r}} \sum_{n=0}^{\infty} e^{\mathrm{i} n \varphi}(\tanh r)^{n} \frac{\sqrt{(2 n) !}}{n ! 2^{n}}|2 n\rangle$ with $\bar{n}_{s}=$ $\sinh ^{2} r=10$. In this case, calculations show that (see the Appendix)

$$
P_{\left(n_{2} ; \xi\right) \rightarrow\left(0 ; \xi, n_{2}\right)}=\frac{P^{n_{2}}{ }_{2} F_{1}\left[\frac{1+n_{2}}{2}, \frac{2+n_{2}}{2}, 1 ; \frac{(1-P)^{2} \bar{n}}{1+\bar{n}}\right]}{\sqrt{1+\bar{n}}},
$$

where ${ }_{2} F_{1}[a, b, c ; z]$ is the hypergeometric function, defined by Pochhammer symbols:

$$
{ }_{2} F_{1}[a, b, c ; z]=\sum_{k-0}^{\infty} \frac{(a)_{k}(b)_{k}}{(c)_{k}} \frac{z^{k}}{k !} .
$$

The dash-dotted black line in Fig. 5 shows the behavior of (13) for $n_{2}=1$ and 10 . The behavior is clearly different from the case of a coherent state: a long plateau occurs. We can conclude that the tunneling probability in the presence of a field in squeezed state $P_{\left(n_{2} ; \xi\right) \rightarrow\left(0 ; \xi, n_{2}\right)}$ is mostly inhibited compared to the case when no field is present in waveguide $B$.

\section{CONCLUSION}

In conclusion we have shown how the tunneling of a single photon as well as multiphoton tunneling can be enhanced or inhibited by the presence of photons. We presented the physical reasons behind such an enhancement or inhibition. A crucial role is played by the bosonic nature of photons. The waveguide structures or fiber couplers are known to be almost decoherence-free; however, if need be then the decoherence effects can be taken into account[15]. Although we explicitly considered the simplest case of a coupler the results can be extended to arrays of couplers. Tunneling is a universal effect in physics; and therefore, the results of this paper would be applicable to all situations in which bosons are involved. Further, the results of this paper should have a bearing on the quantum walk of a single photon in the presence of other photons [16]. The results of this paper should also be applicable to other bosonic systems like cold atoms in multiple traps.

X. L. would like to acknowledge Amanda Taylor for a careful reading of the manuscript.

\section{Appendix: Derivation of Photon Tunneling for Field in Coherent and Squeezed States}

Consider first the case when the field in the waveguide is in a coherent state $|\beta\rangle=\sum_{m=0}^{\infty} c_{m}|m\rangle$ with $c_{m}=$ $e^{-|\beta|^{2} / 2} \frac{\beta^{m}}{\sqrt{m !}}$. Thus the initial state is $\left|n_{2} ; \beta\right\rangle$ instead of $|n, m\rangle$ as considered in Sec. II. The wave function at time $t$ is

$$
|\Psi(t)\rangle=U(t)\left|n_{2} ; \beta\right\rangle=\sum_{m=0}^{\infty} c_{m} U(t)\left|n_{2}, m\right\rangle,
$$

where $U(t)\left|n_{2}, m\right\rangle$ can be obtained from Eqs. (4) - (6). The probability for $n_{2}$ photon tunneling is given by

$P_{\left(n_{2} ; \beta\right) \rightarrow\left(0 ; \beta, n_{2}\right)}=\sum_{l=0}^{\infty}|\langle 0, l \mid \Psi(t)\rangle|^{2}=\sum_{l=0}^{\infty}\left|c_{l}\right|^{2} P_{\left(n_{2}, l\right) \rightarrow\left(0, l+n_{2}\right)}$,

where $P_{\left(n_{2}, n\right) \rightarrow\left(0, n+n_{2}\right)}$ is given by Eq. (8) and hence

$$
P_{\left(n_{2} ; \beta\right) \rightarrow\left(0 ; \beta, n_{2}\right)}=\sum_{l=0}^{\infty}\left|c_{l}\right|^{2}\left(\begin{array}{c}
l+n_{2} \\
n_{2}
\end{array}\right)(1-P)^{l} P^{n_{2}},
$$

The series in Eq. (17) can be summed up, leading to the result (10).

If the field in the waveguide is in the squeezed state $|\xi\rangle=\frac{1}{\sqrt{\cosh r}} \sum_{m=0}^{\infty} e^{\mathrm{i} m \varphi}(\tanh r)^{m} \frac{\sqrt{(2 m) !}}{m ! 2^{m}}|2 m\rangle$, then Eq. (17) holds with $c_{2 l}=\frac{1}{\sqrt{\cosh r}} e^{\mathrm{i} n \varphi}(\tanh r)^{n} \frac{\sqrt{(2 n) !}}{n ! 2^{n}}$ and $c_{2 l+1}=0$. On substituting these values and summing the series we get the result (13).
[1] S. Datta, Electronic Transport in Mesoscopic Systems (Cambridge University Press, Cambridge, 1995); D. K.
Ferry, S. M. Goodnick, Transport in Nanostructures (Cambridge University Press, Cambridge, 1997). 
[2] Mohsen Razavy, Quantum Theory of Tunneling (World Scientific, Singapore, 2003).

[3] H. Grabert and M. H. Devoret, Single Charge Tunneling: Coulomb Blockade Phenomena in Nanostructures, (Plenum Press, New York, 1992).

[4] L. Tian and H.J. Carmichael, Phys. Rev. A 46, R6801 (1992).

[5] A. Imamoğlu, H. Schmidt, G. Woods, and M.Deutsch, Phys. Rev. Lett.79, 1467 (1997).

[6] K. M. Birnbaum et al., Nature (London) 436, 87 (2005).

[7] C. Lang et al., Phys. Rev. Lett. 106, 243601 (2011).

[8] J. B. Williams et al., Phys. Rev. Lett. 87, 037401 (2001); M. Saffman, T. G. Walker, and K. Mølmer, Rev. Mod. Phys. 82, 2313 (2010); J. Gillet, G. S. Agarwal, and T. Bastin, Phys. Rev. A 81, 013837 (2010).

[9] A. Politi et al., Science 320, 646 (2008).

[10] H. B. Perets et al., Phys. Rev. Lett. 100, 170506 (2008); Y. Bromberg et al., ibid. 102, 253904 (2009); S. Longhi, Phys. Rev. A 83, 033821 (2011); Y. Lahini et al., Phys. Rev. Lett. 105, 163905 (2010); Ivan L. Garanovich, Stefano Longhi, Andrey A. Sukhorukov, et al., Phys. Rep.
518, 1 (2012); A. Rai, G. S. Agarwal, and J. H. H. Perk, Phys. Rev. A 78, 042304 (2008).

[11] Jianming Huang and Prem Kumar, Phys. Rev. Lett. 68, 2153 (1992); Ivan S. Grudinin et al., ibid. 104, 083901 (2010).

[12] Alessandro Zavatta, Silvia Viciani, and Marco Bellini, Science 306, 660 (2004); T. B. Pittman, B. C. Jacobs, and J. D. Franson, Opt. Commun. 246, 545 (2005); Sven Ramelow et al., arXiv: 1211.5059v2 (2012).

[13] B. E. A. Saleh and M. C. Teich, Fundamentals of Photonics, (John Wiley \& Sons, New York, 2007), p. 317, Eq. (8-5-6).

[14] R. P. Feynman, The Feynman Lectures on Physics, Vol. 3, (Addison-Wesley, Redwood City, CA, 1989), Sec 21-9.

[15] Amit Rai, Sumanta Das, and G. S. Agarwal, Opt. Express 18, 6241 (2010).

[16] P. K. Pathak and G. S. Agarwal, Phys. Rev. A 75, 032351 (2007); L. Sansoni, F. Sciarrino, et al., Phys. Rev. Lett. 108, 010502 (2012); Alberto Peruzzo et al., Science 329, 1500 (2010). 